\title{
Determination of whether HIPEC is beneficial in patients with synchronous peritoneal and liver metastases from colorectal cancer (Review)
}

\author{
NICOLAE BACALBASA ${ }^{1-3}$, IRINA BALESCU ${ }^{4}$, DRAGOS CRETOIU ${ }^{5,6}$, IOANA HALMACIU $^{7}$, MIHAI DIMITRIU $^{1,8}$, \\ BOGDAN SOCEA $^{9}$, CAMELIA DIACONU ${ }^{10,11}$, LAURA ILIESCU ${ }^{10,12}$, CORNEL SAVU ${ }^{13,14}$, CARMEN SAVU ${ }^{15}$, \\ ALEXANDRU FILIPESCU ${ }^{1,16}$, CLAUDIA STOICA ${ }^{17}$ and OVIDIU STIRU ${ }^{18,19}$ \\ ${ }^{1}$ Department of Obstetrics and Gynecology, 'Carol Davila' University of Medicine and Pharmacy, 020021 Bucharest; \\ ${ }^{2}$ Department of Visceral Surgery, Center of Excellence in Translational Medicine, 'Fundeni' Clinical Institute, \\ 022328 Bucharest; ' ${ }^{3}$ Department of Obstetrics and Gynecology, 'I. Cantacuzino' Clinical Hospital, 030167 Bucharest; \\ ${ }^{4}$ Department of Visceral Surgery, 'Ponderas' Academic Hospital, 021188 Bucharest; ${ }^{5}$ Department of Cellular, Molecular and \\ Histology Biology, 'Carol Davila' University of Medicine and Pharmacy, 020021 Bucharest; ${ }^{6}$ Department of Cellular and \\ Molecular Biology and Histology Scientific Researcher, 'Alessandrescu-Rusescu' National Institute of Mother and \\ Child Health, Fetal Medicine Excellence Research Center, 020395 Bucharest; ${ }^{7}$ Department of Anatomy, \\ ‘George Emil Palade’ University of Medicine, Pharmacy, Science and Technology, 540142 Târgu Mureș; \\ Departments of ${ }^{8}$ Obstetrics and Gynecology, and ${ }^{9}$ Surgery, 'Sf. Pantelimon' Emergency Clinical Hospital, \\ 021659 Bucharest; ${ }^{10}$ Department of Internal Medicine, 'Carol Davila' University of Medicine and Pharmacy, \\ 020021 Bucharest; ${ }^{11}$ Department of Internal Medicine, Clinical Emergency Hospital of Bucharest, 105402 Bucharest; \\ ${ }^{12}$ Department of Internal Medicine, 'Fundeni' Clinical Institute, 022328 Bucharest; ${ }^{13}$ Department of Thoracic Surgery, \\ 'Carol Davila' University of Medicine and Pharmacy, 020021 Bucharest; ${ }^{14}$ Department of Thoracic Surgery, \\ 'Marius Nasta' Institute of Pneumonology, 050159 Bucharest; ${ }^{15}$ Department of Anesthesiology, 'Fundeni' Clinical Institute, \\ 022328 Bucharest; ${ }^{16}$ Department of Obstetrics and Gynecology, 'Elias' Emergency Hospital, 125100 Bucharest; \\ ${ }^{17}$ Department of Visceral Surgery, County Emergency Hospital Ilfov, 022104 Bucharest; \\ ${ }^{18}$ Department of Cardiovascular Surgery, 'Carol Davila' University of Medicine and Pharmacy, \\ 020021 Bucharest; ${ }^{19}$ Department of Cardiovascular Surgery, 'Prof. Dr. C. C. Iliescu' \\ Emergency Institute for Cardiovascular Diseases, 022322 Bucharest, Romania
}

Received April 19, 2021; Accepted May 19, 2021

DOI: $10.3892 / \mathrm{etm} .2021 .10702$

\begin{abstract}
Peritoneal carcinomatosis, as well as the presence of liver metastases from colorectal cancer, has been long considered as the sign of a systemic disease, transforming the patient into a candidate for palliation and best supportive care. However, in recent decades, progress in the field of medical

Correspondence to: Dr Irina Balescu, Department of Visceral Surgery, 'Ponderas' Academic Hospital, 85a Nicolae Caramfil Street, 021188 Bucharest, Romania

E-mail: irina.balescu@ponderas-ah.ro
\end{abstract}

Abbreviations: HIPEC, hyperthermic intraperitoneal chemotherapy; PSOGI, Peritoneal Surface Oncology Group International

Key words: colorectal cancer, peritoneal carcinomatosis, HIPEC, liver metastases, therapeutic procedure and surgical oncology has allowed scientists worldwide to produce curative therapeutic strategies for these cases such as hyperthermic intraperitoneal chemotherapy (HIPEC) or extended liver resection. In addition, the association of these two therapies has also been performed with encouraging results. The aim of the current study was to review articles published thus far in regard to the association of these two therapeutic strategies, in order to identify which cases can benefit the most, which is the most efficient agent or combination of agents, and whether these types of therapy should be performed as monotherapy or as a two-stage procedure.

\section{Contents}

1. Introduction

2. The role of HIPEC in patients with synchronous liver metastases and colorectal cancer

3. Patient selection for HIPEC

4. Two-stage procedure versus one-stage procedure 
5. Types of chemotherapeutic agents used for HIPEC in metastatic colorectal cancer

6. bidirectional HIPEC versus standard HIPEC in metastatic colorectal cancer

7. The role of neoadjuvant therapies in the setting of standard HIPEC

8. Conclusions

\section{Introduction}

Peritoneal carcinomatosis from gastrointestinal primary tumors has been regarded as a sign of advanced stage disease, in which the patient could only be proposed for palliative medical care. In this context, patients with such diseases were usually submitted to centers for best supportive care, their lifespan being limited to a few months; therefore, patients presenting peritoneal or hematogenous metastases from colorectal cancer were reported to have a mean survival rate of 8-10 months (1). However, in recent decades, improvement in the field of surgery and oncology as well as the wide implementation of multidisciplinary onco-surgical meetings have led to the development of new therapeutic strategies (2-5). Therefore, these cases are no longer regarded as candidates for palliative care exclusively and other therapies, such as cytoreduction and the intraperitoneal administration of chemotherapeutic agents, have been proposed. The method currently known as hyperthermic intraperitoneal chemotherapy (HIPEC) has proven to be efficient in cases presenting colorectal cancer and peritoneal metastases, with improved rates of survival being reported at present (6-10). Once these benefits were demonstrated, attention was focused on determining whether this method should be reserved for patients presenting solely with peritoneal metastases or whether it can be extended to cases presenting an association between peritoneal and hematogenous lesions. Among the most intensively debated subjects regarding the association between HIPEC and other visceral resections, that of the association with liver resection is a crucial one.

\section{The role of HIPEC in patients with synchronous liver metastases and colorectal cancer}

The effectiveness and safety of HIPEC in the setting of peritoneal carcinomatosis from colorectal cancer was initially demonstrated in 2003 in a study that was conducted on 105 patients submitted to systemic chemotherapy or cytoreductive surgery and HIPEC. The median survival time was 12.6 months in the standard chemotherapy arm and 22.3 months in the HIPEC arm, the difference being statistically significant $(\mathrm{P}=0.032)(11)$. Five years later the same study group updated the results and reported a disease-specific survival of 21.6 months in the chemotherapy arm and 22.2 months in the HIPEC arm (12); however, it was determined that HIPEC should be reserved for extremely highly selected cases, due to the fact that it may induce a significant degree of perioperative morbidity and mortality. Improvement in the perioperative management of these patients gradually led to a significant decrease in the perioperative rate of complications. In this respect, attention was focused on the possibility of association between cytoreductive surgery and HIPEC and other visceral resections.

Initially it was considered that HIPEC was not indicated in cases presenting liver metastases. However, this hypothesis changed following the first studies that reported acceptable rates of postoperative complications as well as an improved survival rate (11). Therefore, in a study conducted by Elias et al on 24 patients with peritoneal metastases from colorectal cancer submitted to HIPEC, the authors reported a 5-year survival rate of $41.5 \%$ in the absence of liver metastases and $23.6 \%$ in the presence of liver involvement (13). Although apparently the difference between the two values is a significant one, it should be considered that, in the absence of surgical treatment, cases presenting both liver and peritoneal metastases rarely report a longer than 12-month survival (13). A similar conclusion was also demonstrated by the meta-analysis conducted by de Cuba et al (14). Authors of that study underlined the fact that, although patients submitted to debulking surgery, liver resection and HIPEC reported a poorer long-term survival when compared to those submitted to debulking and HIPEC in the absence of liver metastases, their long-term outcomes were significantly improved when compared to cases submitted to standard systemic chemotherapy (14).

Another interesting study conducted on this aspect was published by Tan et al, who demonstrated that the outcomes of patients submitted to cytoreductive surgery and HIPEC are similar to those reported by patients submitted to liver resection for colorectal liver metastases, and therefore concluded that a similar surgical approach should be taken into consideration in cases presenting such types of distant metastases (15).

In due course, more extended studies came to demonstrate that performing cytoreductive surgery, including liver resection, and HIPEC does not increase morbidity while the long-term outcomes reported an improved long-term survival when compared to standard chemotherapy (16-20).

The most important studies conducted on the issue of combined cytoreductive surgery, liver surgery and HIPEC in the setting of metastasis to the liver, peritoneal carcinomatosis, and colorectal cancer are summarized in Table I.

As observed from the aforementioned data, the aim of examining the association between cytoreduction, HIPEC and liver resection was to improve the long-term outcomes of these patients, encouraging surgeons worldwide to include this therapeutic approach in their armamentarium (21-24). As for the preferred agent for HIPEC, this tended to change from mitomycin to oxaliplatin alone or was combined with 5 fluorouracil (bidirectional HIPEC); in this respect certain authors suggested routine replacement of mitomycin with oxaliplatin, albeit this option is currently being debated $(25,26)$. However, irrespective of the type of chemotherapeutic agent, it can be observed that the rates of perioperative deaths remained acceptable while long-term survival was significantly improved.

\section{Patient selection for HIPEC}

As expected, the most important prognostic factors which seem to influence the long-term outcomes of these patients are related to the extent of the disease and to the completeness of the surgical approach. 


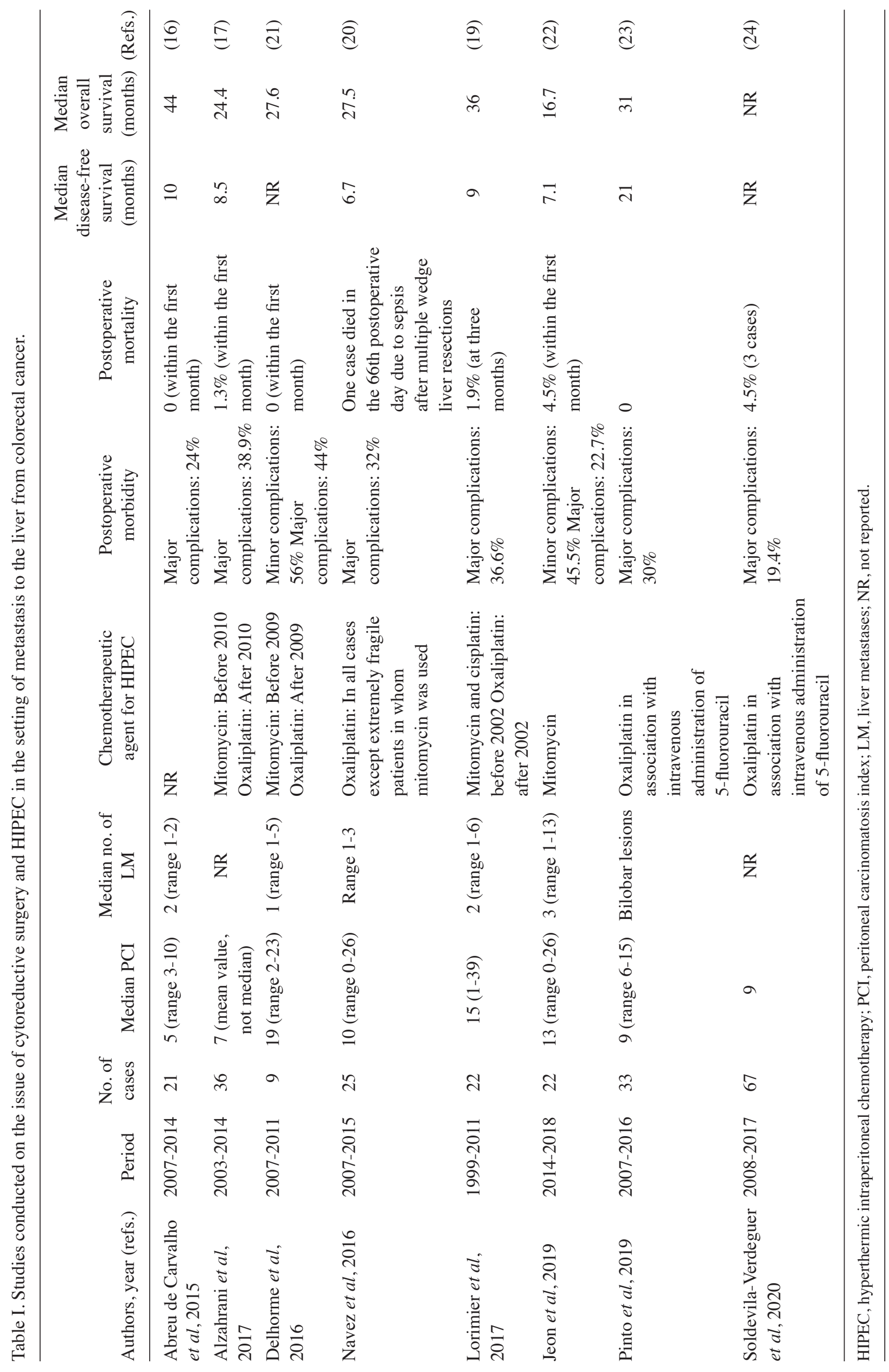


Due to the fact that in this particular category of patients, two pathogenic mechanisms of spread are incriminated (the hematogenous one which is responsible for the development of liver metastases, and the peritoneal one which is responsible for the apparition of peritoneal carcinomatosis), it is evident that each type of spread should be discussed and analyzed in detail. Peritoneal carcinomatosis is expected to be found in up to $15 \%$ of cases at the time of initial diagnosis; moreover, up to $50 \%$ of cases will experience peritoneal recurrence at a certain time point in their disease $(27,28)$. Additionally, up to $45 \%$ of patients diagnosed with colorectal cancer are likely to develop liver metastases at a certain time point in their life (29). Therefore, both situations can be commonly encountered in advanced stage or relapsed disease simultaneously. Concerning peritoneal involvement, this parameter is best evaluated via the peritoneal carcinomatosis index (PCI) (based on the protocol of Sugarbaker) (5). Once the value obtained during peritoneal exploration increases, the likelihood of successful treatment is proportionally decreased; therefore, in peritoneal carcinomatosis from colorectal cancer it has been demonstrated that cases having a PCI value $\leq 10$ are expected to benefit most from HIPEC, while in cases in which PCI is $\geq 20$ the benefit is a minimal one, transforming the intervention into a palliative one $(30,31)$. However, the degree of differentiation of the tumor should be also taken into account; therefore, patients presenting well-differentiated neoplasms are expected to benefit from an increased survival even in the context of a PCI $\geq 10$, and even $\geq 20$, while cases presenting poorly differentiated tumors are expected to have a poor response at $\leq 10 \mathrm{PCI}$ (32).

Regarding PCI values and the likelihood of successful treatment Hentzen et al (33) introduced a hypothesis, delta PCI, which seems to be a more reliable tool in order to identify cases that may benefit most from debulking and HIPEC. Authors of that study considered all patients as potential candidates for debulking and HIPEC. Consequently, all the patients were initially submitted to laparoscopy in order to evaluate the PCI. Furthermore, cases in which laparoscopic evaluation determined that debulking surgery and HIPEC were feasible, were submitted to cytoreductive surgery by an open approach after a median time of one month. Notably, at the time of the open approach certain cases presented a significantly higher value of PCI (the difference between the two values being identified as delta PCI). This difference was explained by Hentzen et al (33) using two theories: i) Biological aggressiveness, a hypothesis whereby in a very short period of time tumoral cells with a higher biological aggressiveness demonstrated a significant explosion at the level of the peritoneal cavity, and ii) the hypothesis regarding visibility: During the open approach visibility and the possibility of exploration of the peritoneal cavity was higher, and therefore, a more accurate evaluation of the peritoneal cavity could be performed. Irrespective of the incriminated theory, the authors demonstrated that a higher delta PCI value was inversely correlated with the likelihood of achieving a long-term survival rate. The authors concluded that cases presenting a delta PCI value $\geq 10$ should be rather referred to the medical oncologist than to cytoreductive surgery and HIPEC; however, no significant correlation could be found between the presence and number of liver metastases and delta PCI (33).
In 2014, Elias et al (34) demonstrated that, not only PCI itself but also the location of the tumoral peritoneal lesions should be taken into consideration when establishing the indication for debulking surgery and HIPEC. In other words, cases presenting a PCI value $\geq 15$ should be considered as having a relative contraindication for debulking and HIPEC, whereas the presence of distal ileal involvement should be considered a negative prognostic factor with regard to long-term outcomes. That study was conducted on 139 patients and reported a 5-year overall survival of $48 \%$ for cases with PCI $<15$ and of only $12 \%$ in cases presenting $\mathrm{PCI} \geq 15$. In addition, involvement of the distal ileum was associated with a 5-year overall survival of $12 \%$, while cases in which this segment was not affected a median overall survival rate of $70 \%$ was reported (34). As expected, there was no significant correlation between the PCI values, the distal ileal involvement and the presence of synchronous liver metastases. Furthermore, association of liver resection did not negatively influence long-term outcomes, demonstrating once again the benefits of association of the two procedures (34).

Liver metastases with colorectal origin are more commonly encountered when compared to peritoneal carcinomatosis. Once the surgical technique for hepatic resection improved, surgical treatment became the option of choice whenever complete resection was expected (35-41). In this respect, it is currently expected that $50 \%$ of patients presenting liver involvement may benefit from complete resection (42). The benefits in terms of survival have been demonstrated worldwide, and a significant improvement of the long-term outcomes was reported (42). However, it seems that the most efficient results were obtained in cases presenting $\leq 3$ liver lesions and a maximum diameter $\leq 3 \mathrm{~cm}$; therefore, this special subcategory of patients was expected to benefit most from hepatic resections. In addition, patients presenting a significant response to neoadjuvant chemotherapy also seemed to have improved long-term outcome after liver surgery (43). Therefore, of importance for this special category of patients (presenting limited extent of liver disease and good response to neoadjuvant chemotherapy) was whether they could be considered good candidates for HIPEC, whenever peritoneal carcinomatosis was encountered (44).

One of the first consensus statements previously published on the issue of peritoneal carcinomatosis and other distant metastases stated that these cases should receive the best chemotherapeutic and biologic agents. Furthermore, patients who report a favorable response to this therapy should be considered for cytoreductive surgery and HIPEC (45).

During a survey conducted by the Peritoneal Surface Oncology Group International (PSOGI) in 2018, HIPEC experts from 19 countries were interviewed with regard to their experience in the field of debulking surgery and HIPEC for colorectal cancer (46). When it came to the presence of liver metastases, a single expert considered that the presence of liver involvement should be considered a formal contraindication for debulking and HIPEC, while 7 experts considered that the presence of $\geq 3$ metastases should exclude the patients from HIPEC and cytoreduction, while the remaining 11 experts considered that the absolute number of liver metastases is not an exclusion criterion. Therefore, the experts recommended 
performing the procedure with curative intent in all cases in which all liver metastases are resectable, as long as complete debulking is achievable (46).

The extent of liver involvement was also intensively debated during one of the most recent consensus conferences conducted on the subject. Accordingly, a maximum number of three liver metastases was considered reasonable in order to further submit the patient to HIPEC and liver resection (47). In addition, other authors consider that a supplementary condition should be considered, that all three lesions be peripheral ones and be located in the same liver lobe. Therefore, Vassos and Piso indicated that, the presence of deeply situated, bilobar or more than three lesions should be considered a formal contraindication for debulking surgery and HIPEC (48).

Completeness of cytoreduction is another factor to be considered in the long-term outcomes of these patients. Therefore, the aim in such cases is to obtain maximal debulking surgery with no visible tumoral lesion in order to remove all the old malignant cells and to improve the intraperitoneal distribution and effect of the chemotherapy agent. However, the histopathological subtype as well as the degree of differentiation should also be taken into account (5).

In their attempt to analyze which patients benefit most from this combined therapeutic approach Maggiori et al included 37 patients with peritoneal and liver metastases who were matched to 61 patients presenting peritoneal metastases exclusively (49). Their findings showed that the optimal outcomes in terms of survival were reported in patients with PCI values $\leq 12$ and no liver metastases (median overall survival of 76 months) followed by patients presenting $\leq 12$ PCI values and 1-2 liver metastases (median overall survival of 40 months) and by those with PCI values $\geq 12$ and $\geq 3$ liver metastases (with a median overall survival of only 27 months). Those authors concluded that cases with PCI values $\geq 12$ and $\geq 3$ liver metastases should not be considered as candidates for surgery with curative intent due to the lower long-term benefits (49). A similar conclusion was published by Downs-Canner et al in 2017 in a study that included 32 patients submitted to combined debulking surgery, HIPEC and liver resection, and 173 patients submitted solely to HIPEC and debulking surgery. Their results demonstrated that simultaneous resection of liver metastases should be avoided if more than three liver lesions are identified (50).

Markers for patients diagnosed with colorectal cancer and undergoing HIPEC, are represented by molecular markers including KRAS and BRAF (51). In a recent study conducted at the Uppsala University, Sweden, patients presenting BRAF mutation tended to have a poor response to debulking surgery and HIPEC. Consequently, the aggressive surgical approach is not recommended in such cases. Furthermore, there was no significant difference in terms of survival between cases presenting KRAS mutations and KRAS wild-type, respectively, enabling the authors to consider that KRAS status should not further influence the decision of performing debulking surgery and HIPEC (51). Similar rates of long-term survival for patients presenting RAS mutations and RAS wild-type, respectively, were also reported in the study conducted by Morgan et al in 2019 (52). However, authors of that study underlined the fact that RAS mutation was associated with significantly poorer disease-free intervals after debulking and HIPEC when compared to wild-type cases (52).
In order to investigate whether age is a formal contraindication for performing such an aggressive procedure, the Spanish study group led by Cascales-Campos performed an analysis on 36 patients, $\geq 75$ years of age, who were submitted to debulking surgery and HIPEC for peritoneal carcinomatosis from colorectal cancer. Of the 36 cases, the authors reported a rate of major complications of $14 \%$ as well as a postoperative mortality rate of $5.4 \%$, values that may be deemed acceptable when compared to those reported in younger groups of patients (53). Concerning the type of chemotherapeutic agent employed for intraperitoneal administration, oxaliplatin was used in $36 \%$ of cases, followed by mitomycin in $33 \%$ of cases, mitomycin in association with doxorubicin in $19 \%$ of cases and irinotecan in $11 \%$ of cases. Association of liver resections with hepatic surgery was $8 \%$ of cases and consisted of minor hepatectomies. By contrast, results of the univariate analysis revealed the association of liver surgery and hepatic metastases with a significantly poorer long-term survival, enabling the authors to consider that in this subgroup, the age association with liver surgery should be carefully analyzed (53).

\section{Two-stage procedure versus one-stage procedure}

Although the effectiveness and safety of liver resection as part of cytoreductive surgery followed by HIPEC has been widely demonstrated, certain authors went even further and investigated whether two-stage procedures can offer any supplementary benefit in terms of reducing the perioperative complication rates such as length of surgery, length of hospital stay, need for reoperation and postoperative morbidity and even mortality rate $(21,50,54)$. Findings of one of the largest studies demonstrated that the two-stage procedure may be beneficial in order to decrease the rates of perioperative complications (54). Cloyd et al included 1,168 patients who underwent cytoreductive surgery and HIPEC, 100 of whom also required liver resections. The authors reported that cases in which cytoreductive surgery and HIPEC were associated with hepatectomy patients had a longer operative time, a longer hospital stay, a higher number of associated surgical procedures and a higher rate of postoperative complications. However, the association of hepatectomy did not increase postoperative mortality (54). Another noteworthy study which came to underline the necessity of two-stage procedures in the setting of colorectal cancer with liver and peritoneal involvement was published in 2016 by Delhorme et al (21). That study included 77 cases submitted to surgery for liver metastases from colorectal cancer, 18 cases submitted to cytoreductive surgery and HIPEC for peritoneal carcinomatosis from colorectal cancer, and 9 cases submitted to cytoreductive surgery, liver resection and HIPEC. The long-term outcomes revealed a median overall survival of 27.6 months for patients who underwent liver surgery, debulking and HIPEC; 39.1 months for patients who underwent debulking and HIPEC; and 52.8 months for cases that underwent surgery for liver metastases from colorectal cancer. In addition, the 2-year survival rates were 78,67 and $78 \%$, respectively, for patients with liver metastases and HIPEC vs. cases undergoing HIPEC and subsequently subjected to liver resection only. However, when it came to the rates of higher than grade III complications, as per 
the Clavien-Dindo classification scale (55), $44 \%$ of patients underwent debulking, HIPEC and liver resection; $11 \%$ underwent debulking and HIPEC, respectively, and $20 \%$ of those underwent liver resection only (21).

Pinto et al demonstrated that the presence of bilobar metastases should no longer be considered a contraindication for debulking, HIPEC and liver surgery; however, in such cases the authors recommended a two-step procedure in order to maximize the effect of surgery without increasing the perioperative risks of severe complications (23).

Another type of sequencing which seems to increase the likelihood of achieving complete debulking surgery in patients presenting extended liver lesions consists of portal vein ligation followed by cytoreductive surgery (including liver resection) and HIPEC. The method seems to be efficient especially in cases presenting more than three hepatic lesions; therefore, in the study conducted by Jeon et al, the authors reported that 6 of the 22 patients underwent liver resection, cytoreduction and HIPEC and benefitted from a two-stage liver approach; of these six cases the number of liver metastases varied between 3 and 11 lesions (22).

\section{Types of chemotherapeutic agents used for HIPEC in metastatic colorectal cancer}

Initially the most commonly investigated regimen for HIPEC was the one represented by mitomycin $(17,19,21)$. Although the benefits reported by the use of mitomycin in association with HIPEC seemed significant at that time period, progress reported in the field of systemic oncological treatment as well as the introduction of oxaliplatin- and irinotecan-based regimens came to decrease the popularity of mitomycin-based HIPEC. One of the most significant studies, which led to this modification in the therapeutic armamentarium of advanced stage colorectal cancer, was conducted by Baratti et al (56). The study included 48 cases administered oxaliplatin/irinotecan perioperative systemic chemotherapy followed by cytoreductive surgery and simultaneously 48 controls were administered the same regimen of perioperative systemic chemotherapy followed by cytoreductive surgery and mitomycin-based HIPEC. The two categories of patients were similar in regard to the extent of the peritoneal lesions; the long-term outcomes reported a median overall survival rate of 39.9 months in the non-HIPEC group and 34.8 months in the HIPEC group $(\mathrm{P}=0.7902)$. In addition, the rates of perioperative complications were similar between the two groups, i.e., concerning the presence of the liver metastases, they were reported and resected in 7 cases in the HIPEC group and in 4 cases in the non-HIPEC group (the difference not being statistically significant) (56).

However, due to the improvement in the field of systemic therapy and due to the wide introduction of FOLFOX (leucovorin calcium, folinic acid, 5-fluorouracil and oxaliplatin) and FOLFIRI (leucovorin calcium, calcium folinate, 5-fluorouracil and irinotecan) regimens, attention was focused on the possibility of using oxaliplatin or irinotecan for intraperitoneal administration during HIPEC. Initially, administration of heated oxaliplatin at the level of the peritoneal cavity was associated with increased hematological toxicity due to the high rates of absorption (which may reach
$68 \%$ after administration within $30 \mathrm{~min})(57,58)$. However, further studies demonstrated that oxaliplatin can be safely administered in the peritoneal cavity. The first data that confirmed the efficacy of these agents were published by the French study groups conducted by Elias et al (59) and Quenet et al (60).

Although the initial trend was to routinely modify the therapeutic strategy and to replace mitomycin-based HIPEC with the oxaliplatin one, certain studies failed to demonstrate the absolute superiority of platinum-based chemotherapeutic agents; therefore, in certain cases the long-term outcomes failed to demonstrate significantly improved survival after administration with oxaliplatin $(25,26)$. A recent study that demonstrated that oxaliplatin and mitomycin are in fact associated with similar long-term outcomes, is that by Bakkers et al (26). The study included 297 patients subjected to debulking surgery and mitomycin/oxaliplatin-based chemotherapy for peritoneal carcinomatosis from colorectal cancer between 2014 and 2017 (26). Authors of that study demonstrated that 1-, 2 and 3 -year survival rates as well as the median overall survival rates were similar between the two groups. Specifically, the reported median overall survival rate for the mitomycin group was of 30.7 and 46.6 months for the oxaliplatin group. Additionally, the number of cases presenting visceral, extraperitoneal disease was similar between the two groups, the single significant difference being related to tumor location (patients undergoing mitomycin-based HIPEC presented more frequently right-sided tumors). Therefore, the authors concluded that no preferred drug for intraperitoneal administration could be advised for such cases; thus, further studies are required to achieve improved personalization of this treatment (26). A recent systematic review focusing on mitomycin versus oxaliplatin in peritoneal metastases from colorectal cancer emphasized the fact that there was no significant difference with regard to long-term outcomes. Nevertheless, patients administered oxaliplatin-based chemotherapy tended to have a higher rate of postoperative complications (61).

In this respect, attention was focused on molecular studies which aimed to identify potential pharmacogenetic biomarkers that might predict the efficacy of mitomycin-based HIPEC. The study by Hulshof et al (62) was conducted in The Netherlands on 253 patients who underwent debulking surgery and mitomycin-based HIPEC and was based on the hypothesis that, in order to be effective, mitomycin requires a metabolic activation by the enzymes NQO1*2, NQO1*3 and POR 28 ; therefore, the authors suspected that cases presenting a low activity of these enzymes may yield a poor response to mitomycin-based HIPEC. The authors observed that the peritoneal recurrence rate at three and six months was significantly higher among cases presenting the NQO1*3 heterozygous/homozygous group; therefore the rates of disease-free survival were significantly lower among patients presenting the NQO1 3 variant allele. However, this difference did not lead to a significant difference in terms of survival. In addition, no correlation was identified between NQO1*2 or POR*28 polymorphisms and the disease-free survival rate/peritoneal recurrence rate. In this respect the authors concluded that personalized therapies (according to the status of NQO1*3) should be taken into consideration for peritoneal carcinomatosis from colorectal cancer (62). 


\section{Bidirectional HIPEC versus standard HIPEC in meta- static colorectal cancer}

In order to maximize the effects of chemotherapy and to minimize the side effects of oncological treatment, the role of bidirectional HIPEC was investigated. The study of Glockzin et al (63) included 190 patients who underwent cytoreductive surgery and HIPEC at the University Hospital Regensburg for peritoneal carcinomatosis for colorectal and appendiceal neoplasms. In all cases intravenous 5-fluorouracil was administered approximately $30 \mathrm{~min}$ prior to the intraperitoneal administration of oxaliplatin and irinotecan, respectively. The results of that study showed that, in both groups there were also patients who necessitated liver resections in order to maximize the debulking effort: One case in the oxaliplatin group and four cases in the irinotecan group. The short-term outcomes reported similar rates of perioperative morbidity and mortality, while the long-term analysis revealed similar rates of survival at 3 years between the two groups (oxaliplatin versus irinotecan). However, a better trend for long-term survival was observed in patients administered oxaliplatin-based HIPEC. In addition, no complication related to liver surgery was encountered, demonstrating in this way the possibility of safe association of hepatic surgery for both regimens of HIPEC during bidirectional therapy (63).

\section{The role of neoadjuvant therapies in the setting of stan- dard HIPEC}

In order to maximize the response to cytoreductive surgery and HIPEC, the question of whether neoadjuvant treatment should be taken in consideration was addressed. Administration of neoadjuvant regimens was expected to decrease the tumoral volume and even to convert unresectable disease to resectable lesions, even in the presence of synchronous peritoneal and hematogenous metastases (including liver lesions) (64). Moreover, the response to neoadjuvant chemotherapy was expected to provide a more optimal selection of cases which should be further submitted to cytoreductive surgery and HIPEC. Consequently a good response to neoadjuvant chemotherapy should be considered a sign of a less biologically aggressive tumor, and therefore candidates for surgery with curative intent should be selected from this particular subcategory (65). However, this hypothesis could not be demonstrated by Waite and Youssef who, after conducting a systematic review, suggested there is no evidence that administration of neoadjuvant chemotherapy may improve the long-term outcomes after cytoreductive surgery and HIPEC in metastatic colorectal cancer (66). That article included 16 studies, 10 of which included patients with liver metastases at the time of initial diagnosis (66).

When it comes specifically to cases diagnosed with colorectal cancer with peritoneal carcinomatosis and liver metastases, attention should also be focused on the safety of association of bevacizumab. It has been widely demonstrated that administration of bevacizumab in association with standard chemotherapy agents significantly improves the outcomes of cases diagnosed with colorectal cancer liver metastasis (67). Consequently, bevacizumab has become part of the standard therapeutic protocol for this specific subcategory of patients (68). Notably, due to its direct effect against vascular endothelial growth factor, bevacizumab significantly increases the risks of developing ischemic complications. Therefore, it has been recognized that bevacizumab administration in the setting of the neoadjuvant therapeutic strategy may increase the risk of postoperative complications (69). Moreover, in a study conducted on 182 patients subjected to cytoreductive surgery and HIPEC it was found that in the group in which the neoadjuvant therapy consisted of bevacizumab administration in association with chemotherapy, the rates of postoperative morbidity were double when compared to the group in which the neoadjuvant treatment consisted solely of chemotherapy (70). However, association of liver resection at the time of cytoreductive surgery and HIPEC did not significantly influence the rates of major postoperative morbidity. Thus, authors of that study recommended that a thorough investigation on the necessity of administration of bevacizumab in the setting of neoadjuvant therapy is crucial, especially in cases in which debulking and HIPEC are carried out as a two-step procedure (70).

\section{Conclusions}

Reported data have demonstrated that cytoreductive surgery and HIPEC in peritoneal carcinomatosis from colorectal cancer is an effective procedure that will help increase disease-free and overall survival even in the presence of liver metastasis. However, in such cases success of the procedure is strongly correlated with the extent of the disease, as well as with the completeness of cytoreduction. Therefore, the method seems to be utilized in cases with lower PCI values and in the presence of not more than three liver metastases. Concerning the type of chemotherapeutic agent that is the most efficient, although it has been considered that oxaliplatin is superior to mitomycin, recent studies have failed to demonstrate the universal superiority of oxaliplatin. Thus, selection of the agent should be carefully personalized in each case. As for the type of approach (one stage versus two-stage procedure) it seems that cases presenting a more extensive disease or a poorer biological status may benefit more if a two-stage procedure is performed in order to reduce the perioperative risks of complications.

\section{Acknowledgements}

Not applicable.

\section{Funding}

No funding was received.

\section{Availability of data and materials}

Not applicable.

\section{Authors' contributions}

$\mathrm{NB}, \mathrm{IB}, \mathrm{OS}$ and DC designed the study. IB, CIS and AF wrote the article. IH, MD, BS, CD, LI, ClS and CaS, collected the data. $\mathrm{NB}$, OS offered scientific advice. DC and CoS revised the manuscript. NB was the supervisor. All authors critically revised the manuscript, approved the final version to be published. 


\section{Ethics approval and consent to participate}

All information provided in this review is documented by relevant references.

\section{Patient consent for publication}

Not applicable.

\section{Competing interests}

The authors declare there are no competing interests.

\section{References}

1. Sammartino P, Sibio S, Biacchi D, Cardi M, Mingazzini P, Rosati MS, Cornali T, Sollazzo B, Atta JM and Di Giorgio A: Long-term results after proactive management for locoregional control in patients with colonic cancer at high risk of peritoneal metastases. Int J Colorectal Dis 29: 1081-1089, 2014.

2. Sugarbaker PH: New standard of care for appendiceal epithelial neoplasms and pseudomyxoma peritonei syndrome? Lancet Oncol 7: 69-76, 2006.

3. Sugarbaker PH, Turaga KK, Alexander HR Jr, Deraco M and Hesdorffer M: Management of malignant peritoneal mesothelioma using cytoreductive surgery and perioperative chemotherapy. J Oncol Pract 12: 928-935, 2016.

4. Sugarbaker PH and Kwong ML: Regional chemotherapy: Possibilities for prevention and treatment of peritoneal metastases from gastric cancer. In: Management of Gastric cancer SMGroup. https://smjournals.com/ebooks/management-ofgastric-cancer/chapters/MGC-16-05.pdf. Access date?

5. Sugarbaker PH: Prevention and treatment of peritoneal metastases: A comprehensive review. Indian J Surg Oncol 10: 3-23, 2019.

6. Pestieau SR and Sugarbaker PH: Treatment of primary colon cancer with peritoneal carcinomatosis: Comparison of concomitant vs delayed management. Dis Colon Rectum 43: 1341-1348, 2000.

7. Tentes AA, Spiliotis ID, Korakianitis OS, Vaxevanidou A and Kyziridis D: Adjuvant perioperative intraperitoneal chemotherapy in locally advanced colorectal carcinoma: Preliminary results. ISRN Surg 2011: 529876, 2011.

8. Noura S, Ohue M, Shingai T, Kano S, Ohigashi H, Yano M, Ishikawa O, Takenaka A, Murata K and Kameyama M: Effects of intraperitoneal chemotherapy with mitomycin $\mathrm{C}$ on the prevention of peritoneal recurrence in colorectal cancer patients with positive peritoneal lavage cytology findings. Ann Surg Oncol 18: 396-404, 2011.

9. Glehen O, Kwiatkowski F, Sugarbaker PH, Elias D, Levine EA, De Simone M, Barone R, Yonemura Y, Cavaliere F, Quenet F, et al: Cytoreductive surgery combined with perioperative intraperitoneal chemotherapy for the management of peritoneal carcinomatosis from colorectal cancer: A multi-institutional study. J Clin Oncol 22: 3284-3292, 2004.

10. Sugarbaker PH, van der Speeten K, Stuart OA, Chang D and Mahteme H: Patient- and treatment-related variables, adverse events and their statistical relationship for treatment of peritoneal metastases. In: Cytoreductive Surgery and Perioperative Chemotherapy for Peritoneal Surface Malignancy. Textbook and Video Atlas. Sugarbaker PH (ed). Cine-Med Publishing, Woodbury, NY, pp183-206, 2012.

11. Verwaal VJ, van Ruth S, de Bree E, van Sloothen GW, van Tinteren $\mathrm{H}$, Boot $\mathrm{H}$ and Zoetmulder FA: Randomized trial of cytoreduction and hyperthermic intraperitoneal chemotherapy versus systemic chemotherapy and palliative surgery in patients with peritoneal carcinomatosis of colorectal cancer. J Clin Oncol 21: 3737-3743, 2003.

12. Verwaal VJ, Bruin S, Boot $H$, van Slooten $G$ and van Tinteren $H$ : 8-year follow-up of randomized trial: Cytoreduction and hyperthermic intraperitoneal chemotherapy versus systemic chemotherapy in patients with peritoneal carcinomatosis of colorectal cancer. Ann Surg Oncol 15: 2426-2432, 2008.

13. Elias D, Benizri E, Pocard M, Ducreux M, Boige V and Lasser P: Treatment of synchronous peritoneal carcinomatosis and liver metastases from colorectal cancer. Eur J Surg Oncol 32: 632-636, 2006.
14. de Cuba EM, Kwakman R, Knol DL, Bonjer HJ, Meijer GA and Te Velde EA: Cytoreductive surgery and HIPEC for peritoneal metastases combined with curative treatment of colorectal liver metastases: Systematic review of all literature and meta-analysis of observational studies. Cancer Treat Rev 39: 321-327, 2013.

15. Tan GH, Teo MC, Chen W, Lee SY, Ng DW, Tham CK and Soo KC: Surgical management of colorectal peritoneal metastases: Treatment and outcomes compared with hepatic metastases. J Gastrointest Cancer 44: 170-176, 2013.

16. Abreu de Carvalho LF, Scuderi V, Maes H, Cupo P, Geerts B, Van Bockstal M, Gremonprez F, Willaert W, Pattyn P, Troisi R, et al: Simultaneous parenchyma-preserving liver resection, cytoreductive surgery and intraperitoneal chemotherapy for stage IV colorectal cancer. Acta Chir Belg 115: 261-267, 2015.

17. Alzahrani N,Ung L, Valle SJ,Liauw W and Morris DL: Synchronous liver resection with cytoreductive surgery for the treatment of liver and peritoneal metastases from colon cancer: Results from an Australian centre. ANZ J Surg 87: E167-E172, 2017.

18. Baratti D, Kusamura S, Iusco D, Cotsoglou C, Guaglio M, Battaglia L, Virzi S, Mazzaferro V and Deraco M: Should a history of extraperitoneal disease be a contraindication to cytoreductive surgery and hyperthermic intraperitoneal chemotherapy for colorectal cancer peritoneal metastases? Dis Colon Rectum 61: 1026-1034, 2018.

19. Lorimier G, Linot B, Paillocher N, Dupoiron D, Verriele V, Wernert R, Hamy A and Capitain O: Curative cytoreductive surgery followed by hyperthermic intraperitoneal chemotherapy in patients with peritoneal carcinomatosis and synchronous resectable liver metastases arising from colorectal cancer. Eur J Surg Oncol 43: 150-158, 2017.

20. Navez J, Remue C, Leonard D, Bachmann R, Kartheuser A, Hubert C, Coubeau L, Komuta M, Van den Eynde M, Zech F and Jabbour N: Surgical treatment of colorectal cancer with peritoneal and liver metastases using combined liver and cytoreductive surgery and hyperthermic intraperitoneal chemotherapy: Report from a single-centre experience. Ann Surg Oncol 23 (Suppl 5): S666-S673, 2016.

21. Delhorme JB, Dupont-Kazma L, Addeo P, Lefebvre F, Triki E, Romain B, Meyer N, Bachellier P, Rohr S and Brigand C: Peritoneal carcinomatosis with synchronous liver metastases from colorectal cancer: Who will benefit from complete cytoreductive surgery? Int J Surg 25: 98-105, 2016.

22. Jeon Y, Park EJ, Lim JH and Baik SH: Clinical outcomes of complete cytoreduction with concurrent liver resection followed by hyperthermic intraperitoneal chemotherapy for synchronous peritoneal and liver metastatic colorectal cancer. World J Surg Oncol 17: 214, 2019.

23. Pinto A, Hobeika C, Philis A, Kirzin S, Carrere N and Ghouti L: Synchronous liver metastases and peritoneal carcinomatosis from colorectal cancer: Different strategies for curative treatment? Langenbecks Arch Surg 404: 477-488, 2019.

24. Soldevila-Verdeguer C, Segura-Sampedro JJ, Pineno-Flores C, Sanchis-Cortes P, Gonzalez-Argente X and Morales-Soriano R: Hepatic resection and blood transfusion increase morbidity after cytoreductive surgery and HIPEC for colorectal carcinomatosis. Clin Transl Oncol 22: 2032-2039, 2020.

25. Quenet F, Elias D, Roca L, Goere D, Ghouti L, Pocard M, Facy O, Arvieux C, Lorimier G, Pezet D, et al: A UNICANCER phase III trial of hyperthermic intra-peritoneal chemotherapy (HIPEC) for colorectal peritoneal carcinomatosis (PC): PRODIGE 7. J Clin Oncol 36 (Suppl 18): LBA3503, 2018.

26. Bakkers C, van Erning FN, Rovers KP, Nienhuijs SW, Burger JW, Lemmens VE, Aalbers AG, Kok NF, Boerma D, Brandt AR, et al: Long-term survival after hyperthermic intraperitoneal chemotherapy using mitomycin $\mathrm{C}$ or oxaliplatin in colorectal cancer patients with synchronous peritoneal metastases: A nationwide comparative study. Eur J Surg Oncol 46: 1902-1907, 2020.

27. Al Shammaa HA, Li Y and Yonemura Y: Current status and future strategies of cytoreductive surgery plus intraperitoneal hyperthermic chemotherapy for peritoneal carcinomatosis. World J Gastroenterol 14: 1159-1166, 2008.

28. Jayne DG, Fook S, Loi C and Seow-Choen F: Peritoneal carcinomatosis from colorectal cancer. Br J Surg 89: 1545-1550, 2002.

29. Grundmann RT: Current state of surgical treatment of liver metastases from colorectal cancer. World J Gastrointest Surg 3: 183-196, 2011.

30. da Silva RG and Sugarbaker PH: Analysis of prognostic factors in seventy patients having a complete cytoreduction plus perioperative intraperitoneal chemotherapy for carcinomatosis from colorectal cancer. J Am Coll Surg 203: 878-886, 2006. 
31. Goere D, Souadka A, Faron M, Cloutier AS, Viana B, Honore C, Dumont $\mathrm{F}$ and Elias D: Extent of colorectal peritoneal carcinomatosis: Attempt to define a threshold above which HIPEC does not offer survival benefit: A comparative study. Ann Surg Oncol 22: 2958-2964, 2015

32. Sugarbaker PH: Pseudomyxoma peritonei and peritoneal metastases from appendiceal malignancy. In: Cytoreductive Surgery and Perioperative Chemotherapy for Peritoneal Surface Malignancy. Textbook and Video Atlas. 2nd edition. Sugarbaker PH (ed). Cine-Med Publishers, Woodbury, NY, pp109-132, 2017.

33. Hentzen JEKR, van der Plas WY, Kuipers H, Ramcharan S, Been LB, Hoogwater FJH, van Ginkel RJ, van Dam GM, Hemmer PHJ and Kruijff S: Delta peritoneal cancer index $(\triangle \mathrm{PCI}):$ A new dynamic prognostic parameter for survival in patients with colorectal peritoneal metastases. Eur J Surg Oncol 46: 590-599, 2020.

34. Elias D, Mariani A, Cloutier AS, Blot F, Goere D, Dumont F, Honore C, Billard V, Dartigues P and Ducreux M: Modified selection criteria for complete cytoreductive surgery plus HIPEC based on peritoneal cancer index and small bowel involvement for peritoneal carcinomatosis of colorectal origin. Eur J Surg Oncol 40: 1467-1473, 2014.

35. BacalbaSa N, Balescu I, Dima S and Popescu I: The role of re-resection for breast cancer liver metastases-a single center experience. Anticancer Res 35: 6877-6880, 2015

36. BacalbaSa N, Balescu I, Dima S and Popescu I: Long-term survivors after liver resection for ovarian cancer liver metastases. Anticancer Res 35: 6919-6923, 2015.

37. BacalbaSa N, Balescu I, Dima S and Popescu I: Long-term survivors after liver resection for breast cancer liver metastases. Anticancer Res 35: 6913-6917, 2015.

38. BacalbaSa N, Dima SO, Purtan-Purnichescu R, Herlea V and Popescu I: Role of surgical treatment in breast cancer liver metastases: A single center experience. Anticancer Res 34 5563-5568, 2014

39. BacalbaSa N, Dima S, Brasoveanu V, David L, Balescu I, Purnichescu-Purtan R and Popescu I: Liver resection for ovarian cancer liver metastases as part of cytoreductive surgery is safe and may bring survival benefit. World J Surg Oncol 13: 235, 2015

40. Bacalbasa N, Brezean I, Anghel C, Barbu I, Pautov M, Balescu I and Brasoveanu V: Successful resection and vascular ligation of a large hepatic artery aneurysm-a case report and literature review. In Vivo 31: 979-982, 2017.

41. BacalbaSa N, Brezean I, Anghel C, Barbu I, Pautov M, Balescu I and Brasoveanu V: Management of a fulminant upper gastrointestinal bleeding exteriorized through hemobilia due to arteriobiliary fistula between the common bile duct and a right hepatic artery aneurysm-a case report. In Vivo 31: 983-989, 2017.

42. Hackl C, Neumann P, Gerken M, Loss M, KlinkhammerSchalke $M$ and Schlitt HJ: Treatment of colorectal liver metastases in Germany: A ten-year population-based analysis of 5772 cases of primary colorectal adenocarcinoma. BMC Cancer 14: 810, 2014.

43. Adam R, Wicherts DA, de Haas RJ, Ciacio O, Levi F, Paule B, Ducreux M, Azoulay D, Bismuth H and Castaing D: Patients with initially unresectable colorectal liver metastases: Is there a possibility of cure? J Clin Oncol 27: 1829-1835, 2009.

44. Khrizman P and Mulcahy MF: Curative approach for stage IV colorectal cancer with multiorgan involvement: What makes sense and what doesn't? Curr Colorectal Cancer Rep 6: 97-107, 2010.

45. Esquivel J, Sticca R, Sugarbaker P, Levine E, Yan TD, Alexander R, Baratti D, Bartlett D, Barone R, Barrios P, et al: Cytoreductive surgery and hyperthermic intraperitoneal chemotherapy in the management of peritoneal surface malignancies of colonic origin: A consensus statement. Society of surgical oncology. Ann Surg Oncol 14: 128-133, 2007.

46. Bushati M, Rovers KP, Sommariva A, Sugarbaker PH, Morris DL, Yonemura Y, Quadros CA, Somashekhar SP, Ceelen W, Dube P, et al: The current practice of cytoreductive surgery and HIPEC for colorectal peritoneal metastases: Results of a worldwide web-based survey of the peritoneal surface oncology group international (PSOGI). Eur J Surg Oncol 44: 1942-1948, 2018.

47. Steffen T, Eden J, Bijelic L, Glatzer M, Glehen O, Goéré D, de Hingh I, Li Y, Moran B, Morris D, et al: Patient selection for hyperthermic intraperitoneal chemotherapy in patients with colorectal cancer: Consensus on decision making among international experts. Clin Colorectal Cancer 19: 277-284, 2020.
48. Vassos N and Piso P: Metastatic colorectal cancer to the peritoneum: Current treatment options. Curr Treat Options Oncol 19: 49, 2018.

49. Maggiori L, Goere D, Viana B, Tzanis D, Dumont F, Honore C, Eveno C and Elias D: Should patients with peritoneal carcinomatosis of colorectal origin with synchronous liver metastases be treated with a curative intent? A case-control study. Ann Surg 258: 116-121, 2013.

50. Downs-Canner S, Shuai Y, Ramalingam L, Pingpank JF, Holtzman MP, Zeh HJ, Bartlett DL and Choudry HA: Safety and efficacy of combined resection of colorectal peritoneal and liver metastases. J Surg Res 219: 194-201, 2017.

51. Graf W, Cashin PH, Ghanipour L, Enblad M, Botling J, Terman A and Birgisson H: Prognostic impact of BRAF and KRAS mutation in patients with colorectal and appendiceal peritoneal metastases scheduled for CRS and HIPEC. Ann Surg Oncol 27: 293-300, 2020

52. Morgan Z, Chow BE, Strong EA, Tsai S, Christians K, Mogal H, Gamblin TC and Clarke CN: RAS mutation status confers prognostic relevance in patients treated with cytoreductive surgery and hyperthermic intraperitoneal chemotherapy for colorectal cancer. J Surg Res 240: 130-135, 2019.

53. Cascales-Campos PA, Lopez-Lopez V, Torres-Melero J, Arjona A, Munoz-Casares FC, Barrios P, Morales R, Pereira F, Bretcha-Boix P, Gonzalez-Bayon L, et al: Survival outcomes in patients aged 75 years and over with peritoneal colorectal carcinomatosis after cytoreductive surgery and hyperthermic intraperitoneal chemotherapy (HIPEC): Multicenter study of the Spanish group of peritoneal cancer surgery (GECOP). Clin Transl Oncol 22: 130-136, 2020.

54. Cloyd JM, Abdel-Misih S, Hays J, Dillhoff ME, Pawlik TM and Schmidt C: Impact of synchronous liver resection on the perioperative outcomes of patients undergoing CRS-HIPEC. J Gastrointest Surg 22: 1576-1584, 2018.

55. Clavien PA, Barkun J, de Oliveira ML, Vauthey JN, Dindo D, Schulick RD, de Santibanes E, Pekolj J, Slankamenac K, Bassi C, et al: The Clavien-Dindo classification of surgical complications: Five-year experience. Ann Surg 250: 187-196, 2009.

56. Baratti D, Kusamura S, Azmi N, Guaglio M, Montenovo M and Deraco M: Colorectal peritoneal metastases treated by perioperative systemic chemotherapy and cytoreductive surgery with or without mitomycin C-Based HIPEC: A comparative study using the peritoneal surface disease severity score (PSDSS). Ann Surg Oncol 27: 98-106, 2020

57. Ferron G, Dattez S, Gladieff L, Delord JP, Pierre S, Lafont T, Lochon I and Chatelut E: Pharmacokinetics of heated intraperitoneal oxaliplatin. Cancer Chemother Pharmacol 62: 679-683, 2008

58. Mahteme H, Wallin I, Glimelius B, Pahlman L and Ehrsson H: Systemic exposure of the parent drug oxaliplatin during hyperthermic intraperitoneal perfusion. Eur J Clin Pharmacol 64 907-911, 2008.

59. Elias D, Lefevre JH, Chevalier J, Brouquet A, Marchal F, Classe JM, Ferron G, Guilloit JM, Meeus P, Goere D and Bonastre J: Complete cytoreductive surgery plus intraperitoneal chemohyperthermia with oxaliplatin for peritoneal carcinomatosis of colorectal origin. J Clin Oncol 27: 681-685, 2009.

60. Quenet F, Goere D, Mehta SS, Roca L, Dumont F, Hessissen M, Saint-Aubert B and Elias D: Results of two bi-institutional prospective studies using intraperitoneal oxaliplatin with or without irinotecan during HIPEC after cytoreductive surgery for colorectal carcinomatosis. Ann Surg 254: 294-301, 2011.

61. Wisselink DD, Braakhuis LLF, Gallo G, van Grevenstein WMU, van Dieren S, Kok NFM, de Reuver PR, Tanis PJ and de Hingh IHJT: Systematic review of published literature on oxaliplatin and mitomycin $\mathrm{C}$ as chemotherapeutic agents for hyperthermic intraperitoneal chemotherapy in patients with peritoneal metastases from colorectal cancer. Crit Rev Oncol Hematol 142: 119-129, 2019.

62. Hulshof EC, Lurvink RJ, Caserta N, de Hingh IHJT, van Wezel T, Bohringer S, Swen JJ, Gelderblom H, Guchelaar HJ and Deenen MJ: Identification of pharmacogenetic biomarkers for efficacy of cytoreductive surgery plus hyperthermic intraperitoneal mitomycin $\mathrm{C}$ in patients with colorectal peritoneal metastases. Eur J Surg Oncol 46: 1925-1931, 2020.

63. Glockzin G, Gerken M, Lang SA, Klinkhammer-Schalke M, Piso P and Schlitt HJ: Oxaliplatin-based versus irinotecan-based hyperthermic intraperitoneal chemotherapy (HIPEC) in patients with peritoneal metastasis from appendiceal and colorectal cancer: A retrospective analysis. BMC Cancer 14: 807, 2014. 
64. Hompes D, Aalbers A, Boot H, van Velthuysen ML, Vogel W, Prevoo W, van Tinteren $\mathrm{H}$ and Verwaal V: A prospective pilot study to assess neoadjuvant chemotherapy for unresectable peritoneal carcinomatosis from colorectal cancer. Colorectal Dis 16: O264-O272, 2014.

65. Klaver YL, de Hingh IH, Boot H and Verwaal VJ: Results of cytoreductive surgery and hyperthermic intraperitoneal chemotherapy after early failure of adjuvant systemic chemotherapy. J Surg Oncol 103: 431-434, 2011.

66. Waite $\mathrm{K}$ and Youssef $\mathrm{H}$ : The role of neoadjuvant and adjuvant systemic chemotherapy with cytoreductive surgery and heated intraperitoneal chemotherapy for colorectal peritoneal metastases: A systematic review. Ann Surg Oncol 24: 705-720, 2017

67. Kabbinavar FF, Hambleton J, Mass RD, Hurwitz HI, Bergsland E and Sarkar S: Combined analysis of efficacy: The addition of bevacizumab to fluorouracil/leucovorin improves survival for patients with metastatic colorectal cancer. J Clin Oncol 23: 3706-3712, 2005.
68. Saltz LB, Clarke S, Diaz-Rubio E, Scheithauer W, Figer A, Wong R, Koski S, Lichinitser M, Yang TS, Rivera F, et al: Bevacizumab in combination with oxaliplatin-based chemotherapy as first-line therapy in metastatic colorectal cancer: A randomized phase III study. J Clin Oncol 26: 2013-2019, 2008.

69. Hapani S, Chu D and Wu S: Risk of gastrointestinal perforation in patients with cancer treated with bevacizumab: A meta-analysis. Lancet Oncol 10: 559-568, 2009.

70. Eveno C, Passot G, Goere D, Soyer P, Gayat E, Glehen O, Elias D and Pocard M: Bevacizumab doubles the early postoperative complication rate after cytoreductive surgery with hyperthermic intraperitoneal chemotherapy (HIPEC) for peritoneal carcinomatosis of colorectal origin. Ann Surg Oncol 21: 1792-1800, 2014. 